Dibattito

\title{
DIECI ANNI DI CODICE ANTIMAFIA - LE MISURE DI PREVENZIONE: BILANCI E PROSPETTIVE*
}

Fabio Basile

Title: Ten years of Antimafia Code. The preventive measures: evaluations and perspectives

\begin{abstract}
The ten-year anniversary of the promulgation of Legislative Decree no. 159 of 2011 - better known as the "Anti-mafia Code" - offers the opportunity for some reflections on the strengths and shortcomings of this legislative text, including its jurisprudential practice. In particular, with reference to one of the subject-matters regulated by this law, namely preventive measures.
\end{abstract}

Keywords: anti-mafia Code, preventive measures, seizure and confiscation, results, perspectives, jurisprudential application

L'anniversario dei dieci anni dalla promulgazione del d.lgs. n. 159 del 2011 - meglio noto come "codice antimafia" - offre l'occasione per alcune riflessioni sui meriti, ma anche sulle criticità di tale testo legislativo e della sua applicazione giurisprudenziale, qui condotte con particolare riferimento ad una delle materie disciplinate da tale testo legislativo: le misure di prevenzione.

Parole chiave: codice antimafia, misure di prevenzione, sequestro e confisca, bilanci, prospettive, applicazione giurisprudenziale

\footnotetext{
* Relazione introduttiva, presentata all'omonimo convegno tenutosi presso l'Università degli Studi di Milano, 29 settembre 2021. Della "Relazione" è stato mantenuto l'andamento discorsivo originario, con l'aggiunta di alcune note a piè pagina.
} 


\section{Introduzione}

L'interesse e l'attenzione per la tematica delle misure di prevenzione risultano ampiamente giustificati dalla grande rilevanza pratica e dall'importanza strategica che tali misure hanno assunto e possono continuare ad avere in futuro per il nostro Paese: come del resto oltre duecento anni fa già scriveva Cesare Beccaria nel suo mirabile libriccino dedicato ai delitti e alle pene, "è meglio prevenire i delitti che punirli: questo è il fine principale di ogni buona legislazione". ${ }^{1}$

In effetti, un ordinamento giuridico che si limitasse alla sola repressione dei reati, rischierebbe di essere un ordinamento che arriva sempre troppo tardi, quando ormai beni giuridici fondamentali del singolo (ad esempio, vita, incolumità) o della collettività (ad esempio, incolumità pubblica, concorrenza e trasparenza dei mercati, corretto agire della P.A., integrità degli ecosistemi) potrebbero risultare irrimediabilmente compromessi.

Il vero quesito non è, quindi, relativo al "se" occorra una prevenzione dei reati, bensì al "come" la medesima debba essere realizzata, e, di certo, l'ideale sarebbe poterla realizzare rimuovendo le cause sociali ed economiche del crimine, attraverso politiche di assistenza sociale e sanitaria, politiche urbane, politiche di istruzione, educazione e avviamento al lavoro.

Nell'ambito dell'odierno convegno, tuttavia, ci "limiteremo" a parlare della prevenzione attuabile attraverso gli strumenti offerti dal d.lgs. n. 159 del 2011, nella ricorrenza del decennale della sua promulgazione: un testo legislativo dagli immensi meriti, che finalmente ha ricondotto in un quadro ordinato e sistematico una pluralità di leggi, che si erano stratificate e aggrovigliate nei decenni precedenti, formando un intrico a tal punto complicato, da risultare quasi inaccessibile agli stessi operatori del diritto.

Certo, il c.d. "codice delle leggi antimafia e delle misure di prevenzione" è tale solo a metà; in particolare, esso non contiene la ricognizione e l'armonizzazione della normativa penale e processuale in materia di criminalità organizzata, pur auspicata dalla relativa legge delega del $2010 .^{2}$ Pertanto, se di “codice" vogliamo parlare,

\footnotetext{
${ }^{1}$ Cesare Beccaria, Dei delitti e delle pene, ed. a cura di G.D. Pisapia, Giuffrè, Milano, 1964, p. 127.

${ }^{2}$ Sulle ragioni di tale opzione autolimitatrice del legislatore delegato, v. Bartolomeo Romano, Il nuovo codice antimafia, in Misure di prevenzione, Sandro Furfaro (a cura di), Utet Giuridica, 2013, p. 45 ss.,
} 
sarebbe più corretto parlare di codice (solo) delle misure di prevenzione; e forse anche la parola "codice" è troppo ambiziosa, trattandosi più che altro di una sorta di testo unico, giacché privo di tratti fortemente innovativi rispetto alla legislazione previgente.

Comunque sia, oggi l'esistenza di un testo fondamentale di riferimento per le misure di prevenzione indubbiamente alleggerisce gli sforzi di individuazione della normativa, rendendone anche più semplice - e, di conseguenza, più frequente l'applicazione da parte dei giudici, e al contempo stimola e agevola la ricostruzione sistematica dell'intera disciplina.

\section{Viaggio nel passato}

Il convegno di oggi - il convegno del decennale - è destinato a trarre bilanci e a individuare prospettive. Il modo migliore per approcciarci a tale compito, allora, forse consiste nel seguire un antico insegnamento talmudico: "se vuoi sapere dove stai andando, scopri prima da dove vieni".

Ebbene, da dove viene l'attuale disciplina delle misure di prevenzione? Per scoprirlo, vi chiedo di accompagnarmi in un viaggio all'indietro nel tempo, e di risalire almeno al 1889, perché è allora che si consumò una sorta di "peccato originale", finora forse non lavato da alcun battesimo.

Il 1889 è, infatti, l'anno della promulgazione del primo codice penale dell'Italia unita, il Codice Zanardelli, un codice liberale, che raccoglieva l'eredità illuminista, e che tra l'altro si emancipava finalmente dai reati destinati a punire gli oziosi, i vagabondi, i mendicanti, le persone "sospette": ${ }^{3}$ un codice moderno - si pensò - deve punire solo "fatti" offensivi di beni giuridici, non "categorie di soggetti".

il quale riporta il parere, fortemente critico, predisposto dalla Commissione Giustizia della Camera dei deputati, nel quale si manifestavano serie perplessità rispetto alla sistemazione, all'interno di un medesimo decreto, anche del diritto penale della criminalità organizzata; operazione che, a giudizio dell'organo parlamentare, avrebbe potuto essere svolta più opportunamente in un separato ed autonomo testo normativo.

${ }^{3} \mathrm{Si}$ consideri che ancora nel codice penale sardo-piemontese del 1859 (artt. $435 \mathrm{ss}$.) nei confronti di oziosi, vagabondi, mendicanti e "persone sospette" (vale a dire, ai sensi dell'art. 447, i "diffamati per crimini o per delitti, e singolarmente per grassazioni, estorsioni, furti e truffe, nonché i "sottoposti alla sorveglianza speciale della Pubblica Sicurezza"), era prevista la pena carceraria. Analoghe incriminazioni erano previste in altri codici italiani preunitari. 
Di certo, tuttavia, non si rinunciò in quell'occasione ad un controllo coercitivo su oziosi, vagabondi, mendicanti, persone sospette, e sugli altri soggetti "pericolosi" per l'emergente società borghese e per l'unità dello Stato appena conquistata: solo che siffatto controllo dalle stanze auree del codice penale venne dirottato nei meandri oscuri della coeva legge di pubblica sicurezza, ${ }^{4}$ così spargendo i germi di un possibile attentato ad alcune libertà fondamentali dei consociati.

A ben vedere, infatti:

1) le categorie dei soggetti pericolosi, destinatari delle misure di prevenzione, risultavano vaghe e imprecise;

2) questi soggetti pericolosi potevano comunque facilmente essere immessi nel circuito del diritto penale, e quindi essere sottoposti a pena, non appena avessero violato una delle innumerevoli prescrizioni loro imposte tramite le misure di prevenzione;

3) soprattutto, un ruolo centrale nella gestione di questo formidabile strumento di limitazione della libertà dei consociati veniva assegnato al potere amministrativo. Questi germi, come è noto, "matureranno" appieno di lì a poco, quando il legislatore fascista ${ }^{5}$ non mancherà di esasperare i tratti autoritari e polizieschi del sistema delle misure di prevenzione. 6

Ma continuando il nostro viaggio nel passato arriviamo finalmente al 1948, alla promulgazione della nostra Costituzione: una Costituzione splendida, "rinnovatrice, progressiva" come la definiva Piero Calamandrei, ma che serbò un sorprendente, e tuttora indecifrabile silenzio sulle misure di prevenzione, nonostante la bruttissima prova di sé che le stesse avevano dato durante il ventennio fascista. ${ }^{7}$

Sotto l'impulso dei primi interventi demolitori della Corte costituzionale urgeva, tuttavia, mettere mano ad una nuova disciplina delle misure di prevenzione: a ciò provvide la legge 27 dicembre 1956, n. 1423, recante “Misure di prevenzione nei

\footnotetext{
${ }^{4}$ Lo stesso giorno, infatti - 30 giugno 1889 - il Parlamento italiano licenziò sia il codice penale, sia la legge di pubblica sicurezza c.d. Crispi.

${ }^{5}$ Il riferimento è, in particolare, al Testo unico delle leggi di pubblica sicurezza del 1926 (r.d. 6 novembre 1926, n. 1848), coordinato nel 1931, tramite il r.d. 18 giugno 1931, n. 773, con il nuovo codice penale Rocco.

${ }^{6}$ In argomento v. Guido Corso, L'ordine pubblico, Il Mulino, Bologna, 1979, p. 262.

7 Edoardo Zuffada, La prevenzione personale ante delictum: alla ricerca di un fondamento costituzionale, in "Diritto penale e Uomo", rivista online, fasc. 9/2021, p. 2 ss.
} 
confronti delle persone pericolose per la sicurezza e per la pubblica moralità". ${ }^{8}$ Questa legge, tuttavia - a parte l'attribuzione di talune competenze all'autorità giudiziaria - non andò molto più in là di una semplice ripulitura della legislazione previgente dai suoi più evidenti e grossolani profili di incompatibilità con i nuovi principi costituzionali, senza apportare significative innovazioni né nella individuazione dei destinatari della prevenzione, né nella concezione di fondo del sistema di prevenzione.

Il fatto che anche il legislatore repubblicano non considerasse le misure di prevenzione un vecchio arnese desueto e inutile, è peraltro confermato dalle successive leggi, che hanno segnato una graduale, continua espansione sia della platea dei possibili destinatari, sia della tipologia delle misure, come avvenuto con la l. 31 maggio 1965, n. 575 (la prima legge antimafia dell'Italia repubblicana).

Proprio la legge n. 575 del 1965 va considerata, nel bene e nel male, davvero fondamentale per la materia che ci occupa, e ciò almeno per tre motivi:

1) perché con essa si intuiscono le enormi potenzialità delle misure di prevenzione quale strumento di contrasto alla mafia;

2) perché a tal fine, inaugurando un precedente poi più volte seguito dal legislatore nei decenni successivi, si crea la prima fattispecie di pericolosità qualificata di epoca repubblicana - quella degli "indiziati di appartenere ad associazioni mafiose" - e lo si fa sfruttando le maglie ben più larghe con cui il principio di legalità si presentava sul fronte della prevenzione rispetto al terreno della repressione penale: siamo nel 1965 quando ancora non esiste il reato di associazione mafiosa. Anzi, il legislatore dell'epoca si ritiene impossibilitato a formulare una fattispecie incriminatrice, precisa, tassativa, di associazione mafiosa: "la mafia non si può definire", si diceva. ${ }^{9}$ E allora cosa fa il legislatore del 1965? “ripiega” sul sistema della prevenzione, dove è sufficiente parlare in termini

\footnotetext{
${ }^{8} \mathrm{Si}$ noti che è solo con la l. n. 1423/1956 che compare nell'ordinamento italiano la locuzione "misure di prevenzione". Prima del 1956, infatti, si discorreva in termini più generici di "misure di polizia" o di "misure preventive".

${ }^{9} \mathrm{Si}$ veda, ad esempio, quanto scriveva in proposito, ancora nel 1976, Pietro Nuvolone, voce Misure di prevenzione e misure di sicurezza, in Enciclopedia del diritto, vol. XXVI, Milano, Giuffrè, 1976, p. 645: "quello della mafia è un fenomeno complesso, non ben definibile: è un modo di vivere, di comportarsi, in virtù di occulti vincoli di solidarietà. E non necessariamente la mafia esprime se stessa attraverso veri e propri delitti. Questa inafferrabilità del fenomeno rende praticamente impossibile una definizione razionale: esso si coglie più che altro a livello intuizionistico, emozionale”.
} 
generici di "indiziato" e di "associazione mafiosa”, senza bisogno di definire né l'uno né l'altra!

3) la legge del 1965 è fondamentale, infine, perché agli indiziati di mafia riserva una disciplina in più punti speciale, ovviamente più rigorosa, rispetto a quella contenuta nella legge "generale" del $1956 .{ }^{10}$

Proseguendo nel nostro viaggio nel passato, nel 1982 - a distanza, quindi, di diciassette anni dalla legge del 1965 - ci imbattiamo di nuovo in una legge antimafia, che a sua volta apporta una rivoluzione nel sistema della prevenzione: con la legge 13 settembre 1982, n. 646 (c.d. legge Rognoni-La Torre), mentre nel codice penale si introduce finalmente l'art. 416-bis, nel sistema della prevenzione si inaugura infatti, la prevenzione patrimoniale, affidata agli istituti del sequestro e della confisca. $^{11}$

Con questa legge si comprende, finalmente - grazie anche alle intuizioni, e al sacrificio, del gen. dalla Chiesa ${ }^{12}$ - che la criminalità organizzata è una "fenomenologia delinquenziale votata, primariamente, all'accumulazione, sistematica e spasmodica, di ricchezza",13 e che pertanto, se la si vuole sconfiggere, occorre primariamente colpirla in questo suo core business.

\footnotetext{
${ }^{10}$ La specialità di tale disciplina era riconoscibile almeno in tre tratti:

- la possibilità di applicare la sorveglianza speciale anche senza la previa diffida (art. 2);

- l'attribuzione del potere di proposta della misura anche ai Procuratori della Repubblica, oltre che al Questore (art. 2);

- soprattutto, la produzione, a seguito dell'applicazione della sorveglianza speciale, di taluni effetti interdittivi, consistenti nella decadenza da determinate licenze, concessioni, iscrizioni ad albi (art. $10)$.

${ }^{11}$ In realtà, alcune limitate ipotesi di confisca ante litteram di prevenzione (ma a dire il vero anche tale qualificazione è controversa) erano previste sia nella legislazione fascista, sia, in via transitoria, nei decreti luogotenenziali emessi subito dopo la caduta del fascismo: sul punto, v. Giuliano Vassalli, La confisca dei beni. Storia recente e profili dommatici, Cedam, Padova, 1951, p. 6 ss.

12 Per un riconoscimento dei grandi meriti del gen. dalla Chiesa (anche) nel contrasto alla criminalità organizzata di tipo mafioso, v. da ultimo i discorsi pronunciati dal Presidente della Repubblica, Sergio Mattarella, e dalla Ministra dell'Interno, Luciana Lamorgese, in occasione del $39^{\circ}$ anniversario della sua uccisione:

https://palermo.repubblica.it/cronaca/2021/09/03/news/mattarella_grazie_a_dalla_chiesa_un_sal to_di_qualita_nella_lotta_alla_mafia_-316328031/

${ }^{13}$ Il virgolettato è tratto dalla motivazione di una sentenza della Cassazione, Sezioni Unite penali, 26 giugno 2014 (dep. 2 febbraio 2015), n. 4880, imp. Spinelli, intervenuta proprio in materia di prevenzione patrimoniale.
} 


\section{Ritorno al presente}

Con un grosso balzo cronologico, e con qualche omissione che spero mi verrà in questa sede scusata, ${ }^{14}$ possiamo ora tornare al presente e, quindi, al nostro codice antimafia: codice di cui all'inizio ho già decantato le tante virtù, ma di cui occorre adesso sottolineare anche alcune criticità.

\subsection{Misure pensate per i mafiosi, ma applicate a tutti?}

La prima e più evidente criticità del codice antimafia del 2011 consiste probabilmente nel fatto che esso - forse più quale esito preterintenzionale, che in forza di un chiaro disegno legislativo - porta a completamento quell'operazione, già avviata "sottotraccia" negli anni precedenti, di estensione indiscriminata a tutti i soggetti pericolosi della disciplina speciale originariamente introdotta solo come strumento di contrasto alla mafia: sintomatica in tal senso è, del resto, la stessa denominazione abituale del d.lgs. n. 159 del 2011 per l'appunto quale "codice antimafia".

Si pensi, in particolare:

- agli effetti interdittivi della sorveglianza speciale - introdotti con la legge antimafia del 1965 per impedire la perniciosa infiltrazione della criminalità mafiosa nell'economia legale - ora definitivamente estesi a tutti i sorvegliati speciali, a prescindere dall'ancoramento della loro pericolosità a reati di mafia o comunque lucrogenetici; 15

- soprattutto, alla confisca: questa confisca rivoluzionaria e dirompente, non più caratterizzata da uno specifico vincolo di pertinenzialità della cosa rispetto al reato, potenzialmente applicabile senza limiti di tempo all'intero patrimonio - basata su solidi fondamenti criminologici e fornita di piena legittimazione politico-criminale

\footnotetext{
14 Per una ricostruzione, sintetica ma completa, della legislazione in materia di misure di prevenzione, vigente prima del 2011, sia consentito rinviare, anche per ulteriori richiami di dottrina, a Fabio Basile, Manuale delle misure di prevenzione - Profili sostanziali (con la collaborazione di Edoardo Zuffada), II ed., Torino, Giappichelli, 2021, p. 9 ss.

${ }^{15}$ In realtà, già la legge c.d. Reale del 1975 aveva esteso tali effetti interdittivi a talune ulteriori categorie di soggetti pericolosi, ma tale previsione negli anni successivi fu più volte oggetto di successivi ritocchi legislativi, talora in senso espansivo, altre volte in senso restrittivo, generando un quadro confuso e incerto, soprattutto in prospettiva intertemporale.
} 
ove impiegata per il contrasto alla mafia - da "confisca antimafia", quale fu originariamente concepita e presentata al pubblico dibattito nel 1982,16 col codice del 2011 viene definitivamente estesa, senza residui e tentennamenti, a tutti i soggetti pericolosi. ${ }^{17}$

Questa omologazione "al rialzo" della disciplina della prevenzione - in qualche modo esacerbata dalla stessa giurisprudenza della Cassazione che per un certo periodo estende a tutti i soggetti pericolosi orientamenti giurisprudenziali in passato formatisi in relazione ai soli indiziati di mafia ${ }^{18}$ - peraltro stride con la marcata eterogeneità delle fattispecie di pericolosità. Una marcata eterogeneità che emerge, a ben vedere, su un duplice fronte:

i) prima di tutto, sul fronte della comparazione tra fattispecie di pericolosità qualificata e fattispecie di pericolosità generica, dal momento che le prime, ormai "tassativizzate" dalla giurisprudenza (sia pur al costo di una loro torsione verso il passato, verso il reato - rectius, i reati già commessi), sono profondamente diverse dalle seconde, le quali hanno ancora contenuti per lo più indeterminati, e per la cui applicazione il giudice, quasi ad libitum, può scegliere se volgere lo sguardo al futuro (al reato di probabile futura commissione), ovvero al passato (al reato di probabile già avvenuta commissione);

ii) in secondo luogo, sul fronte della comparazione delle stesse fattispecie di pericolosità qualificata tra loro, le quali, in realtà, sono profondamente diverse le une dalle altre non solo per l'assai differente tipologia e gravità dei reati di volta in volta richiamati, ma anche, se non soprattutto, per la varietà dei presupposti su cui si fondano:

- per lo più "indizi", sulla falsariga della fattispecie apripista degli "indiziati di appartenere alla mafia”,

\footnotetext{
${ }^{16}$ Tant'è che essa venne innestata nella legge "speciale" del 1965, e non già nella legge "generale" del 1956.

17 Vale, per la confisca di prevenzione, quanto già rilevato sopra, nota 15, per gli effetti interdittivi della sorveglianza speciale: per una dettagliata ricostruzione della relativa disciplina legislativa e della sua evoluzione storica, sia consentito rinviare a Fabio Basile, Manuale delle misure di prevenzione, cit., p. 159 ss.

${ }^{18}$ Si pensi, ad esempio, agli orientamenti giurisprudenziali relativi alla c.d. valutazione autonoma, alla regola dell'inopponibilità dei redditi leciti non dichiarati nella loro integrità e non solo per la quota evasa, nonché alle presunzioni preterlegali di intestazione fittizia, originariamente elaborati dalla Corte di Cassazione nei confronti degli "indiziati di appartenere ad associazioni di tipo mafioso", ma poi estesi - salvo qualche successivo revirement - a tutte le categorie di soggetti pericolosi.
} 
- ma altre volte "atti preparatori, obiettivamente rilevanti, ovvero esecutivi diretti a" realizzare questo o quel delitto;

- o una precedente condanna congiunta alla "proclività" a commettere un reato della stessa specie di quello per il quale si è stati in precedenza condannati;

- o l'aver "fatto parte" di associazione politiche disciolte (senza precisazione se la partecipazione debba risultare da una sentenza di condanna) e l'essere sospettati ("debba ritenersi") di continuare a farne parte;

- oppure, e infine, l'essere "dediti alla commissione" di determinati reati. ${ }^{19}$

Sussiste, pertanto, il timore che il legislatore abbia esteso ad una congerie assai eterogenea di soggetti pericolosi una disciplina molto rigorosa, originariamente pensata per i soli indiziati di mafia, senza adeguatamente verificare la sussistenza dei presupposti socio-criminologici per l'applicazione di questa disciplina anche agli altri soggetti.

\subsection{Altre criticità della disciplina delle misure di prevenzione contenuta nel codice antimafia}

Oltre a quella appena evidenziata (supra, 3.1), anche altre sono le criticità o perlomeno le disfunzionalità del nostro "codice antimafia", che meritano almeno una menzione, sia pur solo a mo' di elenco.

\subsection{1: In relazione alla confisca}

Cominciando dalla confisca e, in particolare, dalla fase della sua applicazione giudiziaria, occorre rilevare che:

1) sempre vive sono le perplessità legate agli standard probatori richiesti ai fini della dimostrazione della "provenienza illecita" e della "sproporzione" dei beni, sebbene la giurisprudenza abbia di recente meritoriamente inserito il limite della c.d. misura temporale della confisca e abbandonato alcuni degli schemi presuntivi più rigorosi e meno fondati a livello empirico, utilizzati invece nel passato;

\footnotetext{
${ }^{19}$ Per una dettagliata documentazione delle affermazioni sopra riportate, sia consentito ancora una volta rinviare - anche per i necessari rinvii a dottrina e giurisprudenza - a Fabio Basile, Manuale delle misure di prevenzione, cit., p. 33 ss.
} 
2) forse ormai maturi potrebbero essere i tempi anche per una rivisitazione dell'orientamento giurisprudenziale che, andando oltre la lettera della legge, preclude l'opponibilità dei redditi non dichiarati al fisco nella loro interezza e non solo per la quota evasa, anche quando si tratti di evasione solo occasionale;

3) de iure condendo, sarebbe altresì auspicabile l'inserimento di una soglia minima di irrilevanza quantitativa della sproporzione (stabilendo, ad es., che la sproporzione inferiore al 10\% del reddito dichiarato è irrilevante), almeno nei casi di gravi e oggettive difficoltà nel fornire la giustificazione documentale dei cespiti patrimoniali: difficoltà che emergono, tra l'altro, quando si deve risalire molto indietro nel tempo, o quando spetta all'erede ricostruire il patrimonio del de cuius "pericoloso".

Se passiamo, poi, alla fase dell'esecuzione della confisca, può osservarsi che:

4) se negli ultimi anni, grazie anche al regolamento dell'Unione europea 2018/1805 passi avanti sono stati compiuti in merito alla confisca transnazionale (destinata ad assumere sempre più rilievo nella prassi perché ad essere transnazionale è prima di tutto la criminalità, in specie quella organizzata ${ }^{20}$ ),

5) ancora fermi a percentuali troppo basse sono, invece, i beni, definitivamente confiscati, non assegnati o non utilizzati: ${ }^{21}$ occorrerà, pertanto, mettere in atto e diffondere buone prassi per il loro riutilizzo, anche per non tradire la fondamentale legge di iniziativa popolare del 1996;

6) c'è poi l'eterno problema della continuità delle imprese, con il suo drammatico risvolto in termini occupazionali, che in tempi di crisi rischia di diventare esplosivo a livello sociale: chiudono le imprese e tanta gente rimane senza lavoro! Il legislatore, negli ultimi anni, ha mostrato, invero, sensibilità a questi problemi, rafforzando tra l'altro gli strumenti della prevenzione patrimoniale c.d. mite, non ablativa, realizzata attraverso l'amministrazione e il controllo giudiziari, ma

\footnotetext{
20 In argomento, volendo, vedi, anche per ulteriori riferimenti di letteratura, Fabio Basile, Il reato di associazione di tipo mafioso e la Convenzione di Palermo, in questa rivista, fasc. 4/2019, p. 74 ss.

${ }^{21}$ Si vedano in proposito i dati comunicati nella Relazione annuale sull'attività svolta dall'Agenzia Nazionale per l'amministrazione e la destinazione dei beni sequestrati e confiscati alla criminalità organizzata per l'anno 2020: https://www.benisequestraticonfiscati.it/anbsc-relazione-anno2020/
} 
purtroppo ha dedicato a questi istituti una disciplina troppo scarna, che la giurisprudenza a fatica (e forse senza averne la titolarità) sta cercando di colmare; 7) la giurisprudenza stessa, del resto, potrebbe valorizzare di più, rispetto a quanto finora fatto, la nuova formulazione dell'art. 20 cod. antimafia, che consente di disporre, al posto del sequestro, l'amministrazione o il controllo giudiziari, che potrebbero rivelarsi - oltre che strumenti di bonifica - prim'ancora formidabili strumenti di indagine per verificare meglio, con più tempo e più risorse, l'eventuale provenienza illecita o sproporzione dei beni.

\subsection{2: In relazione alle misure personali}

Volendo passare ora ad evidenziare alcune criticità inerenti all'attuale disciplina delle misure di prevenzione personali:

1) almeno un cenno va fatto ad avviso orale e foglio di via, di cui si parla pochissimo (anche in sede scientifica), e di cui pochissimo si sa (quanti sono i provvedimenti applicativi? nei confronti di quali tipologie di soggetti sono emanati? quanti di costoro entrano poi nel circuito penale a seguito delle violazioni delle prescrizioni prevenzionali?).22 Il fatto, poi, che la loro applicazione sia affidata al Questore, e il relativo controllo giudiziario al giudice amministrativo, rende alto il rischio di disomogeneità interpretative di quelle stesse fattispecie di pericolosità generica che, ai fini della sorveglianza speciale, vengono invece applicate dal giudice penale: e così, mentre la Cassazione ha ormai "tassativizzato" quelle fattispecie, ancorandole alla previa commissione di reati, ${ }^{23}$ la giurisprudenza amministrativa continua a parlare di "personalità propense" al reato e di "semplici sospetti", prescindendo dal riscontro della previa commissione di reati; ${ }^{24}$

\footnotetext{
22 Di recente, con riferimento, tuttavia, alla sola area milanese, ha colmato questo deficit di conoscenza l'accurata indagine di Elena Mariani, Prevenire è meglio che punire. Le misure di prevenzione personali tra accertamento della pericolosità e bilanciamenti di interessi, Milano, Giuffrè Francis Lefebvre, 2021, p. 395-400.

23 Cfr. Fabio Basile, I pericolosi generici (art. 1, lett. a e b, cod. antimafia): metamorfosi giurisprudenziali, in "Diritto penale e Uomo", rivista online, fasc. 6/2021, p. 5 ss.

${ }^{24}$ Secondo T.a.r. Campania Napoli, sez. V, 19 marzo (dep. 6 giugno) 2019, n. 3083, "l'avviso orale a tenere una condotta conforme alla legge (...) ben può essere motivato con riferimento anche a semplici sospetti a carico del destinatario, purché basati su elementi di fatto che ne facciano ragionevolmente ritenere l'appartenenza ad una delle menzionate categorie ex art. 1": nello stesso senso, T.a.r. Veneto Venezia, sez. III, 27 marzo (dep. 11 aprile) 2019, n. 468; T.a.r. Molise Campobasso,
} 
2) passando alla sorveglianza speciale, dobbiamo poi segnalare la disfunzionalità delle sue prescrizioni "obbligatorie", da applicarsi "in ogni caso" come recita l'art. 8 co. 4 cod. antimafia, anche quando la loro applicazione, nel caso di specie, potrebbero avere effetti desocializzanti (come si può, ad esempio, vietare ad un cameriere, pur colpito da un provvedimento di sorveglianza speciale, di accedere agli esercizi pubblici?), o disfunzionali (che senso ha imporre orari di prolungata permanenza a casa ad un indiziato di truffa aggravata per il conseguimento di erogazioni pubbliche che opera esclusivamente online seduto comodamente sul divano di casa sua?).

Un margine per una personalizzazione delle prescrizioni della sorveglianza speciale parrebbe offerto dalle c.d. prescrizioni facoltative a contenuto libero (art. 8 co. 5, prima parte, cod. antimafia), al prezzo, tuttavia, di ingigantire a dismisura il potere discrezionale - e le responsabilità - del giudice. Forse, de iure condendo, la soluzione migliore potrebbe essere quella di un ampio catalogo legislativo di possibili prescrizioni, una sorta di menù - "prescrizioni à la carte" - all'interno del quale il giudice possa scegliere quelle più adatte alla concreta manifestazione di pericolosità da fronteggiare nel caso di specie;

3) ecco, ho appena pronunciato la parola magica, "pericolosità": fulcro di tutto il sistema delle misure di prevenzione, eppure indeterminata nei contenuti e negli indici di accertamento, e defraudata di qualsiasi seria proiezione prognostica. ${ }^{25}$

sez. I, 7 giugno (dep. 25 luglio) 2012, n. 369; T.a.r. Sicilia Palermo, sez. I, 20 dicembre 2011 (dep. 20 gennaio 2012), n. 156. Inoltre, nella giurisprudenza amministrativa viene costantemente ribadito che "il giudizio sulla pericolosità sociale del soggetto avvisato non richiede la commissione di specifici reati, essendo sufficiente che l'Autorità di polizia sospetti semplicemente della presenza di elementi tali da ritenere la configurabilità, nel soggetto destinatario dell'avviso, di una personalità propensa a seguire particolari comportamenti antigiuridici": T.a.r. Veneto Venezia, sez. III, 27 marzo (dep. 11 aprile) 2019, n. 468; in senso conforme, ex multis: T.a.r. Lazio Roma, sez. I ter, 10 aprile (dep. 24 maggio) 2018, n. 5764; T.a.r. Piemonte Torino, sez. I, 30 aprile (dep. 15 maggio) 2015, n. 796; T.a.r. Puglia Lecce, sez. I, 5 marzo (dep. 10 aprile) 2015, n. 1135; T.a.r. Puglia Lecce, sez. I, 5 marzo (dep. 9 aprile) 2015, n. 1124; T.a.r. Puglia Lecce, sez. I, 5 marzo (dep. 9 aprile) 2015, n. 1123; T.a.r. Campania Napoli, sez. V, 18 dicembre 2014 (dep. 3 febbraio 2015), n. 726. Solo un po' più elevato lo standard quando deve applicarsi il foglio di via: "il provvedimento in questione (...), come da costante giurisprudenza in materia (...), costituendo una misura di polizia diretta a prevenire reati e non a reprimerli, presuppone un giudizio di pericolosità per la sicurezza pubblica il quale, pur non richiedendo prove compiute della commissione di reati, deve essere motivato con riferimento a concreti comportamenti attuali dell'interessato, ossia a episodi di vita atti a rivelare in modo oggettivo un'apprezzabile probabilità di condotte penalmente rilevanti": così, ex multis, T.a.r. Campania Napoli, sez. V, 20 dicembre 2018 (dep. 12 febbraio 2019), n. 775.

${ }^{25}$ Per maggiori approfondimenti in proposito, v. Elena Mariani, Prevenire è meglio che punire, cit., p. 241 ss.

Cross Vol.7 N³ (2021) - DOI: http://dx.doi.org/10.54103/cross-17444 


\section{Sguardo al futuro}

Sul concetto di pericolosità, come pure sulle altre criticità del codice antimafia, sopra menzionate, il discorso potrebbe essere molto lungo: impossibile soffermarvisi ulteriormente in questa sede. Mi sia, allora, solo consentito di formulare in proposito un caveat: non si dica che queste criticità, queste ambiguità interpretative, queste incongruenze normative sono comunque tollerabili perché, in fin dei conti, le misure di prevenzione non sono così afflittive come le pene.

Si tratterebbe, infatti, di un'affermazione che - al di là di difficili operazioni qualificatorie dentro o fuori la matière pénale - è nei fatti spesso infondata, giacché le misure di prevenzione, in concreto, possono assumere contenuti gravemente afflittivi: non è forse gravemente afflittiva l'ablazione definitiva di tutto il patrimonio? anche dei cespiti patrimoniali acquisiti venti, trenta o quarant'anni prima? anche a carico degli eredi? Non è forse gravemente afflittiva la sottoposizione ad una pena, anche detentiva, non appena venga violata questa o quella prescrizione preventiva, quand'anche nella specie si tratti di prescrizione nella in concreto poco ragionevole (si pensi a taluni casi di violazione dell'obbligo di comunicazione delle variazioni patrimoniali), o desocializzante o disfunzionale (si pensi ai precedenti esempi del cameriere e del truffatore online)? Non è, infine, forse gravemente afflittivo un sistema all'interno del quale non vige il divieto di retroattività, dove la ricorribilità in cassazione è molto limitata (nei motivi proponibili e, ancor più, nel termine di soli dieci giorni per la loro proposizione), un sistema che non conosce un equivalente funzionale della prescrizione del reato, che non conosce altre cause di estinzione, né scusanti, né cause di non punibilità, né circostanze attenuanti, né riti abbreviati (istituti che vanno invece moltiplicandosi in sede penale)?

Più che continuare a nasconderci dietro l'ipocrisia della minore afflittività delle misure di prevenzione, converrebbe allora prendere atto che il codice antimafia rappresenta il punto di partenza, non certo il punto di arrivo, di un cantiere ancora aperto (immagine), impegnato a costruire un sistema della prevenzione - sì, certo, efficace - ma anche sostenibile nei costi, umani e patrimoniali, e moralmente, prima ancora che costituzionalmente, legittimo: un cantiere, dove, accanto a quello del legislatore, fondamentale sarà il lavoro di magistrati, forze dell'ordine, avvocati, 
amministratori, che, con la propria competenza e professionalità, in questo ambito forse più che in altri, hanno la possibilità di essere - utilizzando un'espressione cara al beato Rosario Livatino ${ }^{26}$ - non semplici operatori del diritto, ma autentici operatori di giustizia.

\section{Bibliografia}

Basile Fabio, Manuale delle misure di prevenzione - Profili sostanziali (con la collaborazione di Edoardo Zuffada), II ed., Giappichelli, Torino, 2021.

Basile Fabio, I pericolosi generici (art. 1, lett. a e b, cod. antimafia): metamorfosi giurisprudenziali, in "Diritto penale e Uomo", rivista online, fasc. 6/2021.

Basile Fabio, Il reato di associazione di tipo mafioso e la Convenzione di Palermo, in "Cross - Rivista di studi e ricerche sulla criminalità organizzata", fasc. 4/2019.

Beccaria Cesare, Dei delitti e delle pene, ed. a cura di G.D. Pisapia, Giuffrè, Milano, 1964.

Corso Guido, L'ordine pubblico, Il Mulino, Bologna, 1979.

Mariani Elena, Prevenire è meglio che punire. Le misure di prevenzione personali tra accertamento della pericolosità e bilanciamenti di interessi, Giuffrè Francis Lefebvre, Milano, 2021.

Nuvolone Pietro, voce Misure di prevenzione e misure di sicurezza, in Enciclopedia del diritto, vol. XXVI, Giuffrè, Milano, 1976.

Romano Bartolomeo, Il nuovo codice antimafia, in Misure di prevenzione, Sandro Furfaro (a cura di), Utet Giuridica, Torino, 2013.

Vassalli Giuliano, La confisca dei beni. Storia recente e profili dommatici, Cedam, Padova, 1951.

Zuffada Edoardo, La prevenzione personale ante delictum: alla ricerca di un fondamento costituzionale, in "Diritto penale e Uomo", rivista online, fasc. 9/2021.

${ }^{26}$ Cfr. discorso tenuto da Rosario Livatino il 12 settembre 1983, in occasione dei funerali di Elio Cucchiara: https://www.centrostudilivatino.it/livatino-31-anni-dopo-per-essere-magistratioperatori-di-giustizia/ 